\title{
Finding correlations of features affecting energy con- sumption and performance of web servers using the HADAS eco-assistant
}

\author{
Daniel-Jesus Munoz, Mónica Pinto, Lidia Fuentes \\ \{danimg, pinto, 1ff $@$ lcc.uma.es \\ CAOSD, Dpt. LCC, Universidad de Málaga, Andalucía Tech \\ Málaga, Andalucía, Spain
}

Palabras Clave: Energy efficiency $\cdot$ Performance $\cdot$ Web servers $\cdot$ Linux

Lugar de publicación: Springer Computing (2018) 100:1155-1173

Impact factor: 1.654

DOI: https://doi.org/10.1007/s00607-018-0632-7

\begin{abstract}
Resumen(Abstract). The impact of energy consumption on the environment and the economy is raising awareness of "green" software engineering. HADAS is an eco-assistant that makes developers aware of the influence of their designs and implementations on the energy consumption and performance of the final product. In this paper, we extend HADAS to better support the requirements of users: researchers, automatically dumping the energy-consumption of different software solutions; and developers, who want to perform a sustainability analysis of different software solutions. This analysis has been extended by adding Pearson's chi-squared differentials and Bootstrapping statistics, to automatically check the significance of correlations of the energy consumption, or the execution time, with any other variable (e.g., the number of users) that can influence the selection of a particular eco-efficient configuration. We have evaluated our approach by performing a sustainability analysis of the most common web servers (i.e. PHP servers) using the time and energy data measured with the Watts Up? Pro tool previously dumped in HADAS. We show how HADAS helps web server providers to make a trade-off between energy consumption and execution time, allowing them to sell different server configurations with different costs without modifying the hardware.
\end{abstract}

